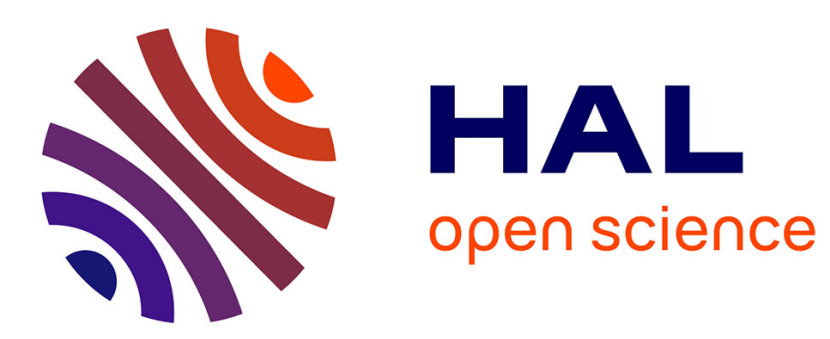

\title{
Quantum beats and polarization interference from excitons in quantum wells
}

\author{
J. Feldmann
}

\section{To cite this version:}

J. Feldmann. Quantum beats and polarization interference from excitons in quantum wells. Journal de Physique IV Proceedings, 1993, 03 (C5), pp.C5-207-C5-214. 10.1051/jp4:1993540 . jpa-00251626

\section{HAL Id: jpa-00251626 https://hal.science/jpa-00251626}

Submitted on 1 Jan 1993

HAL is a multi-disciplinary open access archive for the deposit and dissemination of scientific research documents, whether they are published or not. The documents may come from teaching and research institutions in France or abroad, or from public or private research centers.
L'archive ouverte pluridisciplinaire HAL, est destinée au dépôt et à la diffusion de documents scientifiques de niveau recherche, publiés ou non, émanant des établissements d'enseignement et de recherche français ou étrangers, des laboratoires publics ou privés. 


\title{
Quantum beats and polarization interference from excitons in quantum wells
}

\section{J. FELDMANN}

Department of Physics and Materials Sciences Center, Philipps-University of Marburg, Renthof 5, 35032 Marburg, Germany

\begin{abstract}
We show that time-resolved quantum beat spectroscopy in the four-wave mixing (FWM) geometry is a unique tool to investigate the electronic multi-level system associated with spectrally adjacent excitonic resonances. We have studied heavy- and light-hole excitons, excitons associated with different and spatially separated quantum wells, and excitons associated with spatially separated growth islands within the same quantum well. Though the linear spectra are qualitatively similar in all three cases, the respective beating of the time-resolved FWM-signal shows a very different dynamic behaviour in each case. We discuss appropriate electronic multi-level schemes and show that exciton transitions within different quantum wells can be described by independent twolevel systems. In contrast, excitonic transitions associated with different and spatially separated growth islands having different quantum well thickness are not independent but coupled.
\end{abstract}

In most cases, linear optical spectroscopy does not allow to determine the homogeneous linewidth of excitonic transitions in semiconductor heterostructures, since inhomogeneous broadening caused by interface roughness, alloy disorder, or inhomogeneous electric and magnetic fields contributes to and normally governs the overall linewidth. However, the homogeneous linewidth can be determined by measuring the dephasing time $T_{2}$ of the respective excitonic transition by using nonlinear optical spectroscopy like transient four-wave mixing (FWM) [1]. Important informations about exciton scattering processes in low-dimensional structures can thus easily be obtained. Recently, transient FWM experiments have also been applied to study periodic beating between distinct and simultaneously excited excitonic transitions [2]. The time-period $T_{B}$ of the observed beating is simply the inverse of the spectral spacing between the respective excitonic transitions. Quantum beat spectroscopy in the FWM geometry allows the determination of spectral spacings even when inhomogeneous broadening inhibits to observe the distinct excitonic resonances in the linear optical spectrum $[3,4]$. The possibility to resolve homogeneous linewidths and closely spaced spectral resonances in spite of strong inhomogeneous broadening makes the technique of time-resolved FWM so powerful in the field of low-dimensional structures, where inhomogeneous broadening effects cannot be avoided. 
In this paper, we show that quantum beat spectroscopy in the FWM geometry with temporal resolution of the diffracted signal additionally provides information on the structure of the underlying electronic multi-level system. In particular, it is possible to study whether adjacent excitonic transitions are independent or coupled, i.e., whether excitation of one transition affects the other one or not. Applying this experimental method we find that heavy-hole excitonic transitions associated with spatially separated quantum wells are independent (uncoupled), whereas heavy-hole excitonic transitions associated with spatially separated growth islands within the same quantum well are not independent but coupled.

The set-up for time-resolved FWM experiments is schematically depicted in Fig.1. Laser pulses from a mode-locked Ti-sapphire laser with a pulse width of $110 \mathrm{fs}$ and a spectral width of $21 \mathrm{meV}$ are used for the experiment. Two laser pulses at $t=0$ and $t=\tau$ having wavevectors $k_{1}$ and $k_{2}$, respectively, impinge onto the sample and lead to a diffracted signal into the phase-matched direction $2 k_{2}-k_{1}$. In transient FWM experiments, the total energy of the diffracted signal is measured as a function of time delay $\tau$ using a slow photodetector (not shown in Fig.1). In time-resolved FWM experiments, the diffracted signal is up-converted using a nonlinear crystal and a third reference laser pulse. Thus, the 'real time' behaviour of the nonlinear FWM signal can be measured (for various time delays $\tau$ ) by varying the delay $t$ of the third reference pulse. All experiments presented in this paper are performed at a sample temperature of $T=5 K$.

By time resolving the FWM signal using cross-correlation techniques $[3,5,6]$ or a streak camera [7] it has been shown that excitation of an inhomogeneously broadened excitonic transition leads to the emission of a photon echo (PE). This is expected for an ensemble of independent two-level systems, which statistically differ in energy [8]. For a single two-level system showing a pure homogeneous linewidth a free induction decay (FID) is expected in a time-resolved FWM experiment. It has been shown theoretically $[9,10]$ and experimentally $[11,12]$ that in case of homogeneously broadened excitonic transitions a pure FID is not observed. A delayed increase of the FWM signal is observed and is attributed to many-body Coulomb effects [9-12]. Recently, transient FWM experiments have

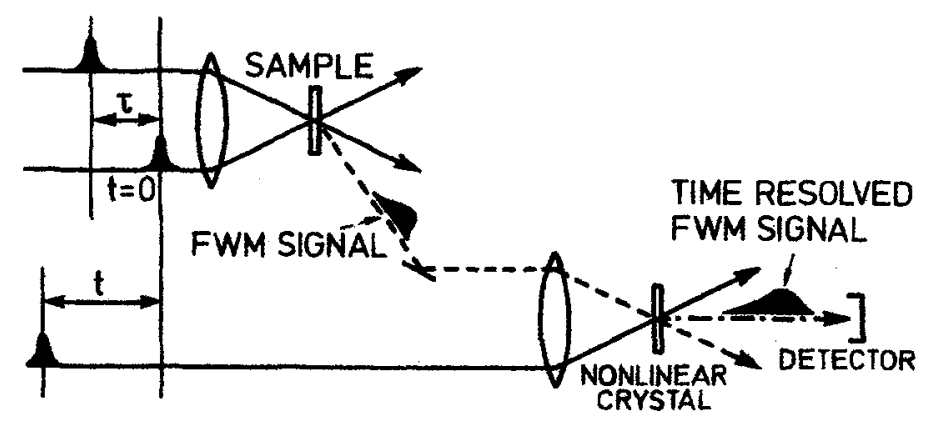

Fig.1: Experimental setup for the time-resolved FWM experiment. 
been used to study quantum beats between different excited excitonic transitions in semiconductor heterostructures $[2,13]$. The broad spectrum of a short laser pulse allows to simultaneously excite spectrally distinct excitonic transitions and the beating behaviour is a consequence of the different transition frequencies of the two excitonic resonances. The time-period $T_{b}$ is connected to the spectral spacing according to

$$
T_{B}=h / \Delta E
$$

The beat signal is discussed in terms of multi-level excitation schemes. The most prominent example is a three-level system (3-LS) as shown on the right side of Fig.2a, which accounts for quantum beats arising from the simultaneous excitation of heavy- and light-hole excitons $[14,15]$. Göbel et al. [16] observed a beating of the transient FWM signal when the distinct excitonic transitions associated with different and spatially separated monolayer islands were simultaneously excited. The authors attributed the observed periodic modulations to quantum beats (QB). Subsequently, it was argued that this oscillatory structure should not be called QB, since it originates from two spatially separated oscillators, i.e., from two independent or uncoupled two-level systems (2x2-LS) as schematically depicted on the right side of Fig.2b. Thereafter, this 'island' beating has been attributed to polarization interference (PI) [2], however, no experimental verification has been possible up to recently. We have shown recently that in fact time-resolved detection of the beating FWM signal allows to decide, which electronic multi-level system properly describes the beating FWM signal $[17,18]$.

Here, we present time-resolved quantum beat experiments performed on three different quantum well samples. The linear optical spectra (absorption spectrum or photoluminescence excitation spectrum) of these samples are shown in Fig.2. Sample A is a GaAs/AlAs multiple quantum well (MQW) structure with a quantum well thickness of $150 \AA$. The corresponding absorption spectrum in Fig.2a shows the $n=1$ heavy-hole (hh) and light-hole (lh) excitonic transitions. Since both transitions share the same $n=1$ electron state in the conduction band, they represent a. 3-LS as indicated on the right side of Fig.2a. Sample B contains two subsequent and thus spatially separated MQW structures, each containing forty wells with a well width of $80 \AA$ and $90 \AA$, respectively. As can be seen in the absorption spectrum of Fig. $2 \mathrm{~b}$, the $n=1 \mathrm{hh}$-excitonic transitions associated with the spatially separated MQWs are energetically close together, whereas both $n=1 \mathrm{lh}$ excitonic transitions are at higher energy. Accordingly, it is possible to tune the optical spectrum of the exciting laser pulse in such a way that only these two hh resonances are excited, which should then represent a $2 \times 2-L S$ as indicated on the right side of Fig.2b. Sample C is the same as in Ref. [16] and consists of ten periods of $70 \AA$ quantum wells grown with growth interruptions of $60 \mathrm{~s}$ at each interface. The PLE spectrum in Fig.2c shows three distinct peaks with an energy splitting of $\Delta E=3.1 \mathrm{meV}$ (after deconvolution of the inhomogeneous lines). This splitting corresponds to a monolayer thickness difference for the $n=1 \mathrm{hh}$ excitonic transition. These excitonic transitions are thus associated with distinct and spatially separated growth islands. Depending on whether two or three excitonic transitions are simultaneously excited, this system should represent a $2 \times 2-$ LS or a $3 \times 2-$ LS as discussed in Ref.[2]. However, we show in the following that only sample B exhibits PI as expected from a $2 \times 2-\mathrm{LS}$, whereas sample $\mathrm{A}$ and $\mathrm{C}$ exhibit QB.

The experimental results of the time resolved FWM experiments are shown in Fig.3a-c for all samples A-C, respectively. The up-converted FWM signals are plotted as a function of both the real time $t$ and the time delay $\tau$. In all three cases periodic modulations of the signal are observed, which correspond to the inverse of (i) the hh-lh splitting for sample A, (ii) the spectral spacing between the 

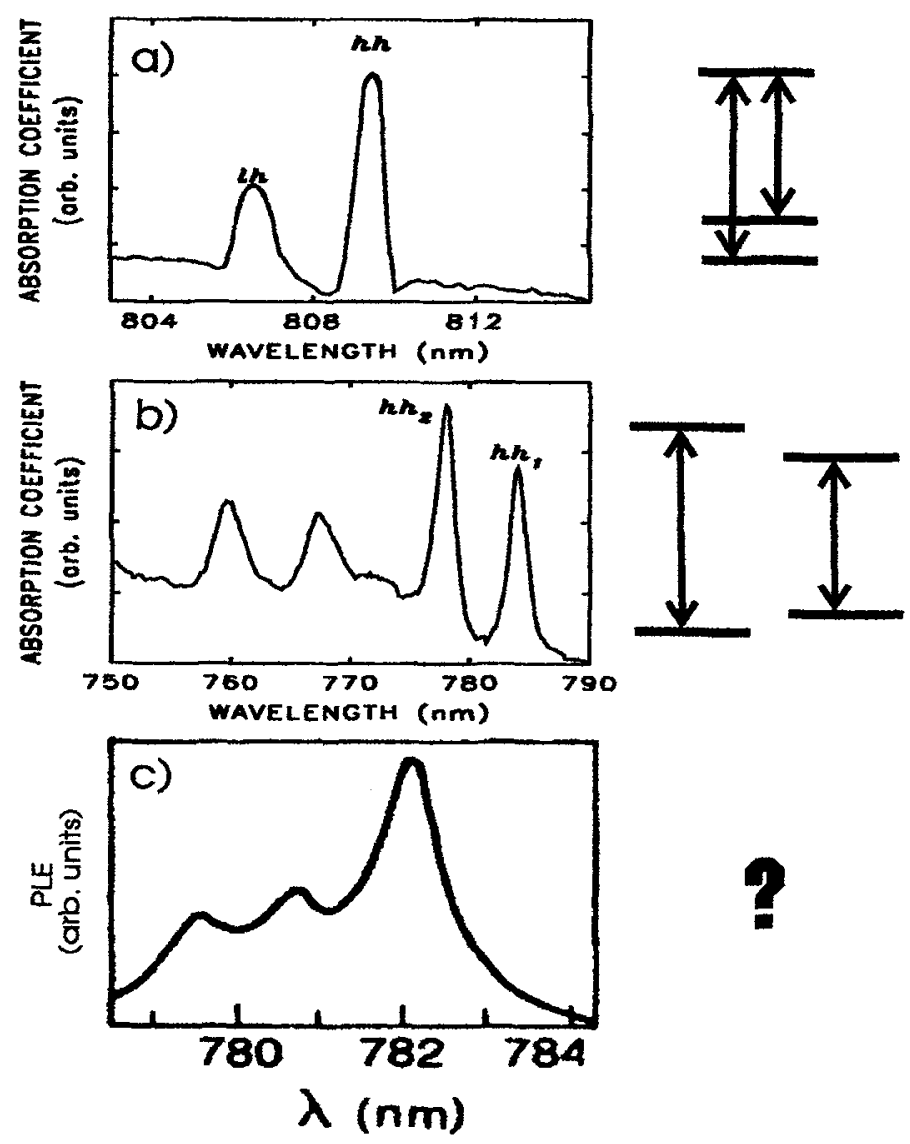

?

Fig.2: Linear optical spectra for samples A-C together with a depiction of the expected electronic multi-level systems.

two $n=1$ hh excitonic resonances for sample $\mathrm{B}$, and (iii) the monolayer induced splitting of the $n=1$ hh excitonic resonance for sample $C$. In order to analyse these data, we have plotted the maxima of the beating FWM signals as a function of real time and time delay in the insets of Fig.3a-c. In case of sample $A$, the experimentally determined positions of the maxima are well described by

$$
t_{\text {max }}=\tau+n \cdot T_{B} \quad \text { (solid lines in Fig.3a) }
$$

with $n=1,2,3,4$. In contrast, the positions of the maxima in case of sample $\mathrm{B}$ closely follow the relationship

$$
t_{\text {max }}=2 \cdot \tau+n \cdot T_{B} \quad \text { (solid lines in Fig.3b) }
$$

with $n=-1,0,1,2$. As was shown recently [17], relations (2) and (3) are indeed theoretically expected for a 3-LS and a 2x2-LS, respectively. Accordingly, time-resolved FWM allows to unambigously 


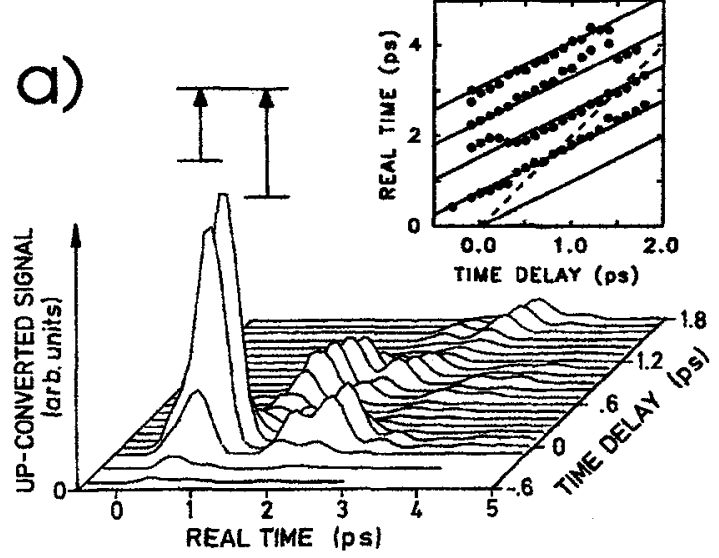

b)
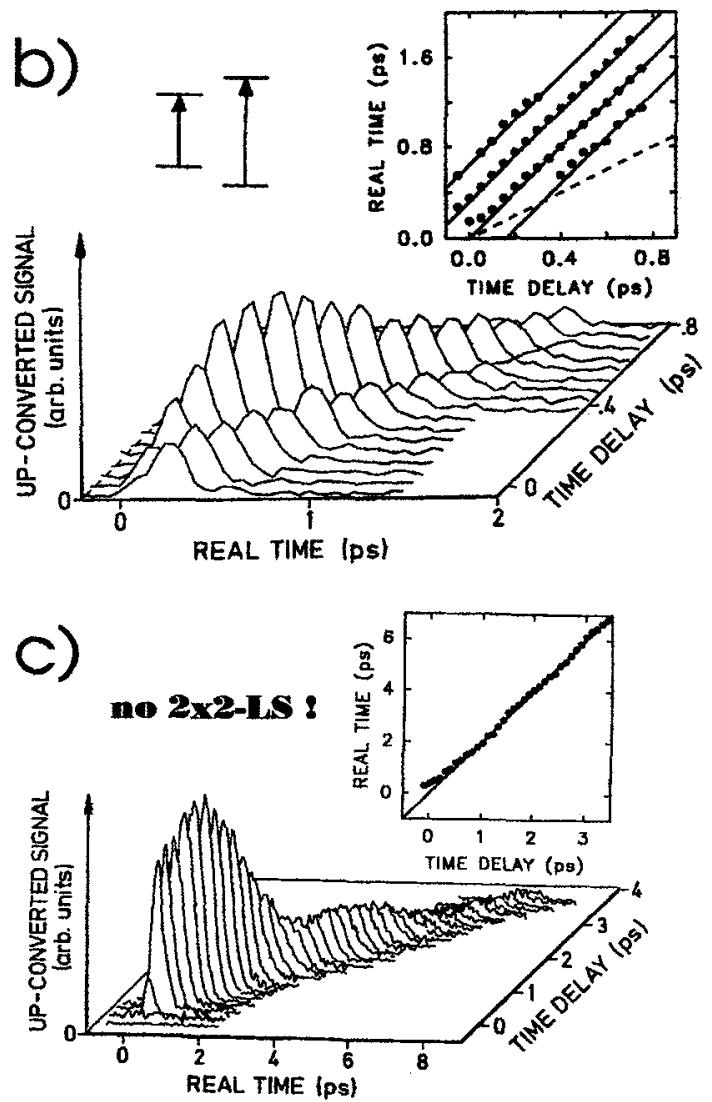

Fig.3: Up-converted FWM signal as a function of time delay $\tau$ and real time $t$ for samples A-C. The positions of the observed maxima are plotted as dots in the insets. The solid lines represent fits as discussed in the text. 
distinguish between a 3-LS which gives rise to QB and a $2 \times 2-L S$ which leads to PI. The fact that the two hh excitonic transitions of sample B behave like a $2 \times 2-\mathrm{LS}$ and show PI means that these two transitions are totally independent, i.e., uncoupled. Excitation of one $\mathrm{hh}$ exciton resonance does not affect the transition frequency, the transition strength or the dephasing rate of the other $\mathrm{hh}$ exciton resonance.

In case of sample $\mathrm{C}$, the time-resolved signal exhibits an unmodulated, nearly symmetric shape with a temporal halfwidth of about $800 \mathrm{fs}$ for each time delay $\tau$. This time-resolved signal can be classified as a 'broad' photon echo, as can be seen more clearly in the inset of Fig.3c, where the positions of the maxima follows the condition

$$
t=2 \cdot \tau \quad \text { (solid line in Fig. } 3 \mathrm{c} \text { ) }
$$

as expected for a photon echo. In contrast to samples A and B, the temporal width of the photon echo signal, which is mainly determined by the inhomogeneous linewidth of the excitonic transitions, is smaller than the beat period. Accordingly, the positions for the maxima plotted in the inset of Fig.3c are a consequence of the photon echo effect and are not determined by a particular beating phenomenon. However, the amplitude of the photon echo exhibits a modulation as a function of the time delay $\tau$ with a time period $T_{B}=1.33 p s$ consistent with the monolayer induced splitting in the linear spectrum of Fig.2c. Comparison of Eq.(3) and (4) shows that a photon echo signal never exhibits a modulation when the underlying multi-level system is a $2 \times 2-L S$, since both the photon echo and the beating of a $2 \times 2-\mathrm{LS}$ simultaneously exhibit maxima at $t=2 \tau$ (see also the experimental verification in Fig.3b). The fact that the photon echo at $t=2 \tau$ exhibits beats as a function of $\tau$ shows that the underlying multi-level system cannot be a $2 \times 2$-LS. This means that the excitonic resonances associated with distinct and spatially separated growth islands within the same quantum well cannot be described by two uncoupled two-level systems. Accordingly, the observed signal beats do not arise from polarization interference but are due to quantum beats as postulated by Göbel et al. [16]. In contrast, the experimental results shown in Fig.3b demonstrate that excitonic resonances associated with spatially separated quantum wells are independent and behave like uncoupled twolevel systems leading to polarization interference. Very recently, Lyssenko et al. [19] found that beats between spectrally adjacent bound exciton resonances in CdSe can also be described by polarization interferences between two non-interacting, i.e., uncoupled two-level systems.

Further understanding of what is meant by 'coupling' can be obtained by considering how coupling modifies the underlying level system. The left-hand side of Fig. 4 illustrates the uncoupled 2x2-LS in the one-particle picture, whereas the right-hand side of Fig. 3 shows the $2 \times 2$-LS in the two-particle picture. In the ground-state $|g\rangle=\mid 00>$ of the crystal none of the excitons are excited. The states $\mid 10>$ and $\mid 01>$ indicate that one of the respective exciton transitions is excited, whereas $\mid 11>$ indicates that both excitons are excited. The calculation of the FWM signal using the right-hand level system leads to the same result, namely PI behaviour, as the calculation using the left-hand one-particle picture provided that transitions $A$ and A' as well as B and B' are identical in terms of transition frequency, transition strength and dephasing rate [20]. The excitation of one excitonic transition then does not affect the other excitonic transition, i.e., the two excitonic transitions are independent or uncoupled. In the opposite case, when excitation of one transition does affect the excitation of the other one, i.e., when $A$ and $A^{\prime}$ or B and $B^{\prime}$ are not identical, the right-hand level-system does not lead to polarization interference but to quantum beats. In this case, the two 
excitonic transitions are not independent and the beating behaviour of the FWM signal can no longer be calculated in the simple one-particle $2 \times 2$-LS. In this sense, the excitonic transitions associated with different and spatially separated quantum well islands are coupled.
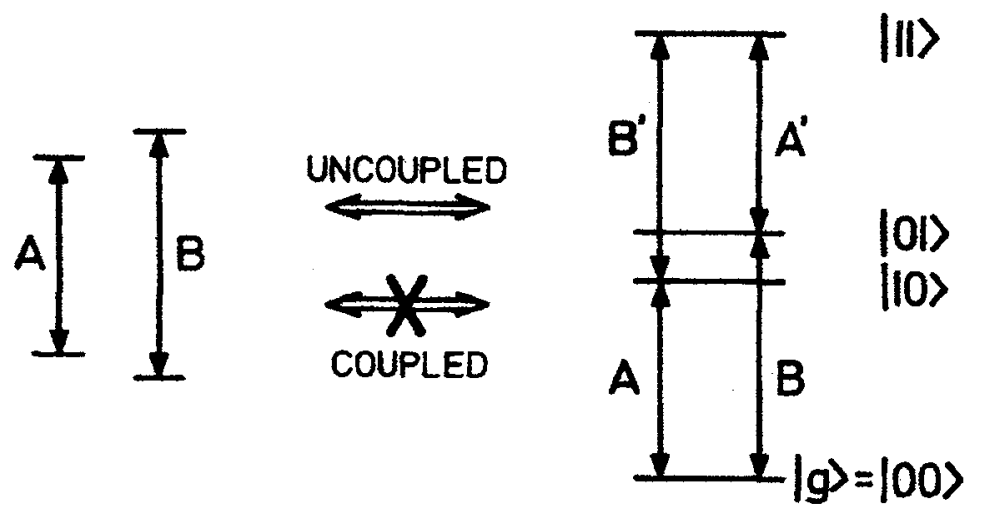

Fig.4: Level scheme for a $2 \times 2$-LS in the one-particle (left side) and the two-particle picture (right side). The equivalency between both pictures only holds in the uncoupled case, where $A=A^{\prime}$ and $B=B^{\prime}$.

There are several possible mechanisms which might lead to a coupling, i.e., to an inequality of the $A$ and $A^{\prime}$ or the $B$ and B' transitions. First of all, the excitation of one excitonic transition might lead to a bleaching of the other transition, since both exciton states might use the same one-particle k-states. Secondly, screening of the Coulomb interaction might lead to a coupling of the two exciton transitions. The coupling may also be caused by Coulomb-exchange interaction, i.e., by manyparticle-Coulomb effects [9-12] and/or by dipole-dipole interaction [21,22]. Further experimental and theoretical work is clearly needed to obtain a definite answer on the origin of the coupling mechanism.

In summary, we have shown that time-resolved detection of the beating FWM signal allows to determine the nature of the electronic multi-level system causing the periodic signal modulations. In particular, the mutual coupling of distinct excitonic transitions, i.e., the perturbation of one transition by the excitation of the other one, can be studied. We find that excitonic transitions associated with different and spatially separated quantum wells can be described by two independent two-level systems, whereas excitonic transitions associated with spatially separated in-plane growth islands cannot and thus must be coupled. We propose bleaching, screening, many-particle Coulomb effects, and dipole-dipole interaction as possible coupling mechanisms.

Acknowledgement: The results reported here have been obtained in cooperation with M. Koch, G. von Plessen, E.O. Göbel, P. Thomas, J. Shah, K. Köhler, S.T. Cundiff, D. Bennhardt, and D. Weber. We thank M. Preis for excellent technical assistance. The work at Marburg University is financially supported by the Deutsche Forschungsgemeinschaft through the Leibniz-Förderpreis. 


\section{References:}

1. see e.g., J. Kuhl, A. Honold, L. Schultheis, and C.W. Tu, Festkörperprobleme/Advances in Solid State Physics, Vol. 31, ed. by U. Rössler, (Vieweg, Braunschweig 1989), p.157, and references therein.

2. K. Leo in 'Optics of Semiconductor Nanostructures', ed. by F. Henneberger, S. Schmitt-Rink, and E.O. Göbel (Akademie Verlag, Berlin 1993), p.127, and references therein.

3. M. Koch, D. Weber, J. Feldmann, E.O. Göbel, T. Meier, A. Schulze, P. Thomas, S. Schmitt-Rink, and K. Ploog, Phys. Rev. B 47, 1532 (1993).

4. T. Albrecht, J.H.H. Sandmann, J. Feldmann, W. Stolz, E.O. Göbel, A. Nebel, C. Fallnich, and R. Beigang, submitted for publication.

5. L. Schultheis, M.D. Sturge, and J. Hegarty, Appl. Phys. Lett. 47, 995 (1985).

6. M.D. Webb, S.T. Cundiff, and D.G. Steel, Phys. Rev. Lett. 66, 934 (1991).

7. G. Noll, U. Siegner, S.G. Shevel, and E.O. Göbel, Phys. Rev. Lett. 64, 792 (1990).

8. T. Yajima and Y. Taira, J. Phys. Soc. Jpn. 47, 1620 (1979).

9. M. Lindberg, R. Binder, and S.W. Koch, Phys. Rev. A45, 1865 (1992).

10. W. Schäfer, F. Jahnke, and S. Schmitt-Rink, Phys. Rev. B47, 1217 (1993).

11. D.S. Kim, J. Shah, T.C. Damen, W. Schäfer, F. Jahnke, S. Schmitt-Rink, and K. Köhler, Phys. Rev. Lett. 69, 2725 (1992).

12. S. Weiss, M.A. Mycek, J.Y. Bigot, S. Schmitt-Rink, and D.S. Chemla, Phys. Rev. Lett. 69, 2685 (1992).

13. J. Feldmann, T. Meier, G. von Plessen, M. Koch, E.O. Göbel, P. Thomas, G. Bacher, C. Hartmann, H. Schweizer, W. Schäfer, and H. Nickel, Phys. Rev. Lett. 70, 3027 (1993).

14. B.F. Feuerbacher, J. Kuhl, R. Eccleston, and K. Ploog, Solid State Commun. 74, 1279 (1990).

15. K. Leo, T.C. Damen, J. Shah, E.O. Göbel, and K. Köhler, Appl. Phys. Lett. 57, 19 (1990).

16. E.O. Göbel, K. Leo, T.C. Damen, J. Shah, S. Schmitt-Rink, and W. Schäfer, Phys. Rev. Lett. 64, 1801 (1990).

17. M. Koch, J. Feldmann, G. von Plessen, E.O. Göbel, P. Thomas, and K. Köhler, Phys. Rev. Lett. 69, 3631 (1992).

18. M. Koch, J. Feldmann, E.O. Göbel, P. Thomas, J. Shah, and K. Köhler, Phys. Rev. B, in press.

19. V.G. Lyssenko, J. Erland, I. Balslev, K.-H. Pantke, B.S. Razbirin, and J.M. Hvam, submitted for publication.

20. S.T. Cundiff, PhD-thesis, University of Michigan 1992.

21. W. Huhn and A. Stahl, phys. stat. sol. b 124, 167 (1984).

22. T. Takagahara, Phys. Rev. B31, 6552 (1985). 